\title{
Virtual Police - To identify who Bend Rules on the Road
}

\author{
Herath H. M. P. M. B \\ Faculty of Computing, Sri \\ Lanka Institute of Information \\ Technology \\ Colombo, Sri Lanka
}

\author{
Fernando A. D. N. M \\ Faculty of Computing, Sri \\ Lanka Institute of Information \\ Technology \\ Colombo, Sri Lanka
}

\author{
Ailapperuma D. C. R \\ Faculty of Computing, Sri \\ Lanka Institute of Information \\ Technology \\ Colombo, Sri Lanka
}

\author{
Ranaraja V. W. R. M. W. B \\ Faculty of Computing, Sri Lanka Institute of \\ Information Technology \\ Colombo, Sri Lanka
}

\author{
Fernando S. G. S \\ Senior Lecturer, Faculty of Computing, \\ Sri Lanka Institute of Information Technology \\ Colombo, Sri Lanka
}

\begin{abstract}
Road accidents and traffic violations are common issue for all the countries. Most of the accidents are caused because of unsafe driving. There are rules and regulations provided by law in order to avoid road accidents and to drive carefully. There are many Information Communication Technology (ICT) applications to detect vehicles that violate the rules. These are not efficient because it does not have evidences or proofs to take a proper action. People nowadays are not tend to drive safe. There are violations such as over speeding, redlight violation and overtaking another vehicle on single and double line violations. In this system a framework to identify who breaks the rules will be detected and take actions according to the rule that has broken. To identify them, image processing technologies, tools and hardware tools such as ultrasonic sensors, raspberry-pi pc and cameras will be used. A key benefit of this system is that it can reduce the number of road violations that can happen and people will be afraid to violate rules because of the automated system. The systems currently available does not support all the features, this system will be covering red light violations, over-speeding and vehicle overtaking violations as well as this system will be using techniques such as background subtraction, object tracing and ultrasonic sensors. "VirtualPolice" system attempts to result reliable and more efficient comparing to systems that are available. This system is designed to collect evidences of the violations and take actions accordingly. When a violation is updated the system is programmed to notify nearest policemen android devices.
\end{abstract}

\section{Keywords}

OpenCV, python, background subtraction, object tracing, red light violations, over-speeding, vehicle overtaking violations, image processing, raspberry-pi pc, raspberry-pi camera, android and ultrasonic sensors.

\section{INTRODUCTION}

Solid In all the countries there are driving rules available for people to drive carefully by being honest and respecting. When these rules are broken it is defined as a road violation. There road violations that happens in day to day traffic. For an example most common violations such as red light violation, over-speeding, overtaking other vehicles through double white lines and single white lines. These road protocols are applied thoroughly for the places where most of the accidents happen.
Research team have implemented an OpenCV and python based system upon a Raspberry-pi PC and have developed an ASP.NET Web API for an android application to alert the nearest police device. This system is named as "VIRTUALPOLICE". This system is implemented to ease the work to the police using a user interface. Image processing technology is used to detect the lanes, vehicles and will identify vehicles who break rules. The team have implemented the system with the ultrasonic sensors in order to system to receive conditions to identify when a violation occur. It will be easier for the police to catch who break laws of the traffic and for the policemen who take bribes and dishonest people will also be reduced. An image of the violation with the location, time, date and an image of the vehicle to the nearest policemen devices by deriving devices around the current location that violation happened.

As an example "When a vehicle speeds and if it cuts the line on a traffic light when it is on red light proposed system will detect them and take photos of the vehicle as evidences according to the protocol that is broke and send an alert to the nearest policemen device" this research is mainly considered about catching who break the rules. This system covers few components such as to detect vehicles who overtake in wrong places, vehicles that speeds and traffic light protocol violations. This system will be more reliable and vehicle detection and protocol identification will be accurate.

\section{BACKGROUND STUDY}

To implement the "Virtual Police" application, resources were gathered from many sources such as similar research papers books documents and magazine articles. A summary of the research results are as follows.

Many quite systems have implemented to detect violations, vehicles and extensive methods of image processing techniques. The research done by Winserng $\mathrm{C}$ states that using Canny Edge Detector, Sobel Operator and Lines Transform are used in this research Portable and compact enough to install in the wind shield. Helps to know how image processing technology is used [1]. "A Real-Time Vision System for Nighttime Vehicle Detection and Traffic Surveillance". Have used loop detectors, slit sensors and raspberry pi cameras to detect and classify vehicles in various night-time environments by Chen Y.L. et al. [2]. "Morphological Image Processing Approach of Vehicle Detection for Real-Time Traffic Analysis" have used 
morphological operations, background subtraction, edge detection and thresholding. Prutha Y.M. and Anuradha S.G. have not used any of sensors, heavy traffics can be identified and low cost but extremely sensitivity to light [3].

Saha S. et al. have used background subtraction. This team was able to have reliable with outdoor lighting conditions and possible vibrations of the camera in order to detect vehicles that violate red lights in India [4]. Cucchiara R. et al. by mainly focusing on vehicle detection and have implemented to detect vehicles day time and night with the use of spatialtemporal and morphological analysis but the team have not able to detect lanes in the road [5]. A research that was done by Coifmana B. et al. were attempted and have researched to how to use video image processing to quest for better traffic information, and thus, an increasing reliance on traffic surveillance, has resulted in a need for better vehicle detection such as wide-area detectors; while the high costs and safety risks associated with lane closures has directed the search towards non-invasive detectors mounted beyond the edge of the pavement where the team was able to detect a wide area while congestion and shadows have been a problem[6]. Jain A., Reddy H. and Dubey $\mathrm{S}$ was able to state with the use of MATLAB for grey scale, threshold and canny edge detector. A research for the vehicle to be automated and that identifies the road signs that are available and drive accordingly even though the system fails to work under different weather conditions and circumstances [7]. Ushma A. et al. have used optical character recognition, $\mathrm{C}++$ and opencv. By localizing license plate regions from true colour images while the efficiency defects on noisy images [8]. A research that was done by Handaric R. and Floroia D. have used MATLAB, image enhancement and edge detection to identify the objects in digital images and size of each object [9]. "Hybrid Parking Detection" by Zhang G. et al. have used background subtraction and edge detection to robust and reliable to identify parking spaces [10].

These researches mostly consider about few components of the road safeness. "VirtualPolice" covers common traffic violations and the system is built to identify who break rules in the road such as over speeding, Red light violation and overtaking on single and double lines. It will be easy and time saving for the police to handle work. The android system facilitates the nearest traffic policemen to take actions for the violation messages that have received.

\section{METHODOLOGY}

The prototype methodology was used to achieve the project goals. Planning, Analysis, Designing and Implementation phases were executed concurrently and iterated until the project reached all the objectives and the users were satisfied with a final prototype. First prototype was built with minimal amount of features and the rest of the features were added to each prototype produced.

\subsection{Planning}

In this step the research team identified the problem correctly. Most of the people does not drive carefully because of the current system to catch who break the rules is not efficient enough to collect any evidences. This system implement to ease the work at police stations by collecting evidences. From the feasibility analysis helped to identify the risks associated with the further proceeding of the system. The project team decided to use Image Processing technologies, Raspberry-pi devices, OpenCV and Python programming skills. In financially also the proposed system is very feasible to develop. The objectives for the system need to list and all the tasks that needed to be accomplished.

- Gathering requirements.

- Identifying the objectives and functions.

- Modelling the web server architecture.

- Designing the web server model.

Work plan of the proposed system is described in work break down structure.

\subsection{Requirement Gathering and Analysis}

After identifying the problem, the next phase is to analyze the problem. In this step the research group analyzed the feasibility of the research, understand the stakeholder's needs and defined the system that will be capable of detecting violations. Main stakeholders are the police who will use "VirtualPolice" to ease their work.

After referring research papers related to the proposed research to identify information which must be gathered and after identify requirements what must be included to proposed research, information must be gathered related to requirements.

\subsection{Design}

This is the high level architecture diagram that was designed by the research group for "Virtual Police".

According to the Fig 1, there are mainly two users who are interacting with the system. Traffic policemen will interact with the android system to check and take actions according to the alert they receive as to forward it to the next nearest policemen, cancel and caught. The details are sent when a violation is detected and store details in the database. Administrator using the web application can create new traffic policemen and check all the details that have currently received from the analysis service in raspberry pi pc and change user account details. The analysis service checks for any vehicles that break the road rules and send the details to the database. All the above-mentioned data are taken from a hosted database and a server hosted in Microsoft azure.

The hardware that needs to accomplish tasks are been sketched in a circuit diagram to ensure a proper integration with the raspberry pi pc such as ultrasonic sensors. Fig 2 above illustrates the hardware circuit diagram sketched by the research group.

As according to the Fig 2 power for module was given using Vcc, ground it using GND, and use our Raspberry Pi to send an input signal to TRIG, which triggers the sensor to send an ultrasonic pulse. The pulse waves bounce off any nearby objects and in some situations the waves are reflected back to the sensor. 


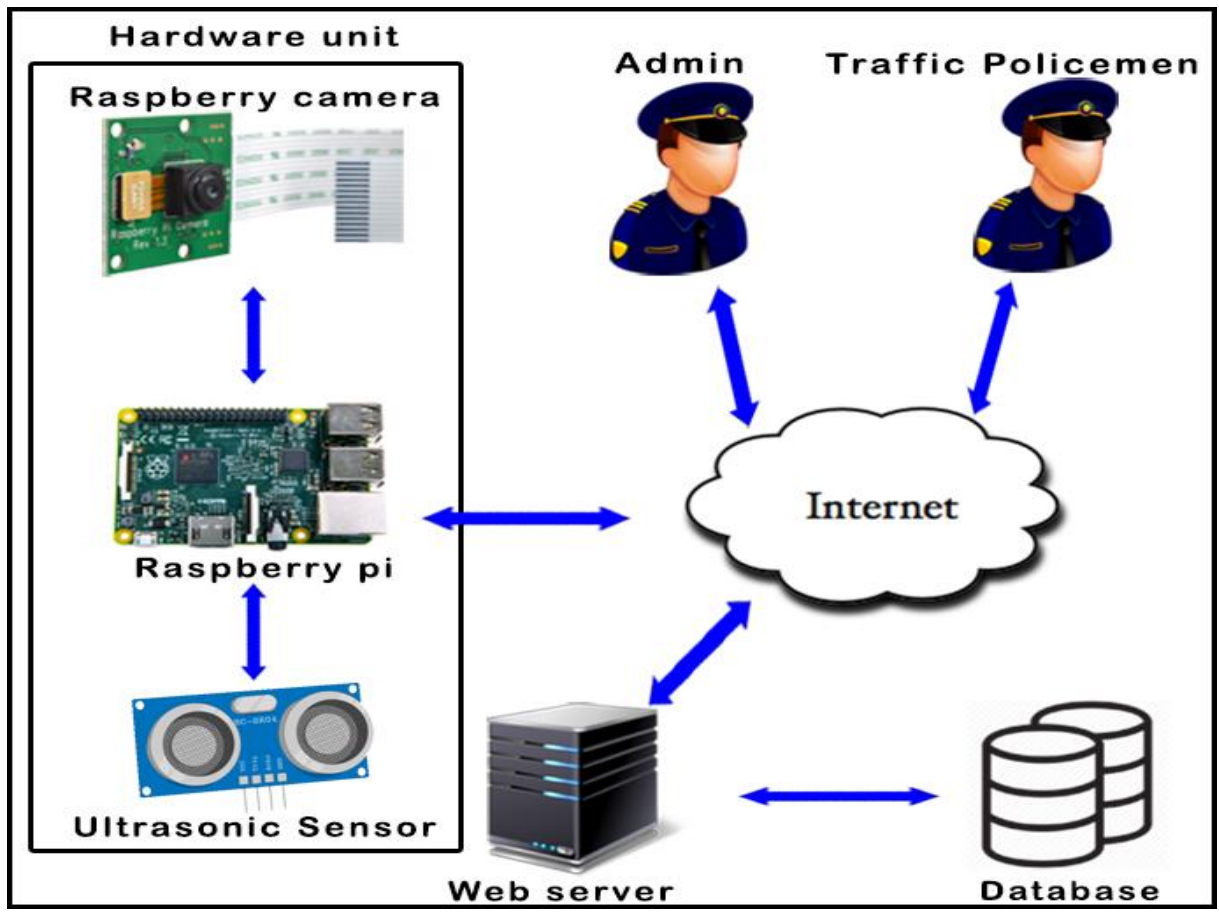

Fig 1: High Level Architecture Diagram

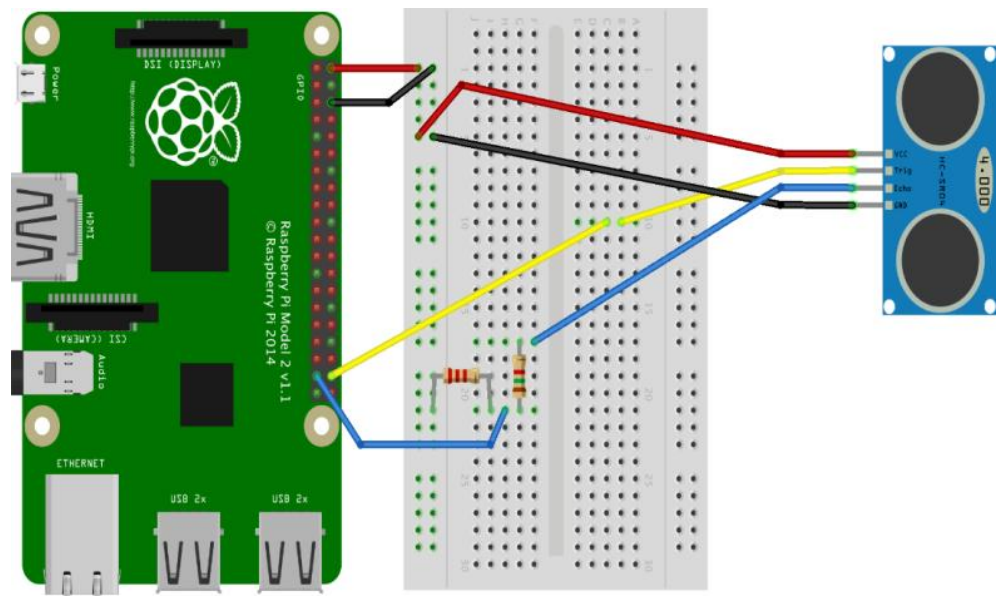

Fig 2: Hardware Circuit Diagram

\subsection{Implementation}

In implementation phase project team have to code and develop the system. After completing the whole system, it is forwarded to the testing phase. In this part the main objective is to code the system. Since a website is included we use JavaScript to code the system to handle all the client side programming. And ASP.NET to handle server side programming. The database connection we are using is MSSQL. The Android application is developed using Android Studio.

The modules that are related are grouped together so that each programmer is working on related program modules and are assigned to programmers to implement. After implementing all modules, they all are integrated to build the final System. Test Plan that defines a series of tests that will be conducted will be prepared. Both System Documentation and User Documentation are prepared before the submission of the final system.
The integrated system was been tested using a model road that was designed by the research group in order to check whether image processing functionalities that is built with the use of ultrasonic sensors works accurately and efficiently as users require.

\section{RESULTS AND DISCUSSIONS}

Virtual Police Analysis Service was developed using image processing techniques and with ultrasonic sensors. In this system there are three main function as, over speeding, detecting while over taking a vehicle, violating the traffic light. In order to identify the over speeding methodology the research group developed a technique using two ultrasonic sensors. Through ultrasonic sensors it calculates by taking the time and distance that takes a vehicle to pass the two sensors. Once the vehicle tend to pass the speed limit, using the raspberry pi camera an image will be captured of the violated vehicle. The methodology which developed to detect the vehicle while overtaking is identified by the raspberry camera. 
The camera will identify the white line which is drawn on the road and if a vehicle overtakes a vehicle by crossing the white line the camera will capture an image instantly. Furthermore as a main component the research group developed a technique to identify the vehicles which violates the traffic lights. To identify the traffic light violated vehicles the raspberry camera and ultrasonic sensors are been used. The system works it task as if the vehicle crosses the white line while the red light is turned on the camera will capture the vehicle which crossed the white line. An ultrasonic sensor is been adapted straight to the white line and when the waves are been distracted the built system identifies that a vehicle have violated.

The captured images which was discussed above will be sent to the database which is hosted in Microsoft Azure using asp.net web API. Along with the image details of the violation will also been sent to the hosted database. Web API identifies the nearest device according to the violated location. Through a given radius the system identifies all the devices within and sends the messages to nearest police devices.

Android application was developed using Android Studio IDE. Application was developed for the use of the traffic policemen. Android application will search for a violations which is nearest to the located traffic policemen. Moreover, through this application it will view the violations through a recycler view. Policemen can take three actions with use of the android application, if the policeman caught the traffic violated vehicle the database will be updated. Another action which the application perform is if in case the policeman could not catch the traffic violated vehicle he can forward it to next nearest policeman by recalling the Web API or he can cancel the received message. All the above mentioned tasks will be updated to the database. Fig 3 and Fig 4 are the screenshots of results that were taken from the android device as in the messages that will be showing to the police user.

The Fig 3 below shows all the messages that have received for a specified device. The android application requests the Web API to send all the messages that are available for the current location of the user and they will be displayed in a recycler view as such. When the user select a message all the details and the action buttons will be displayed as in Fig 4 in order to take actions.

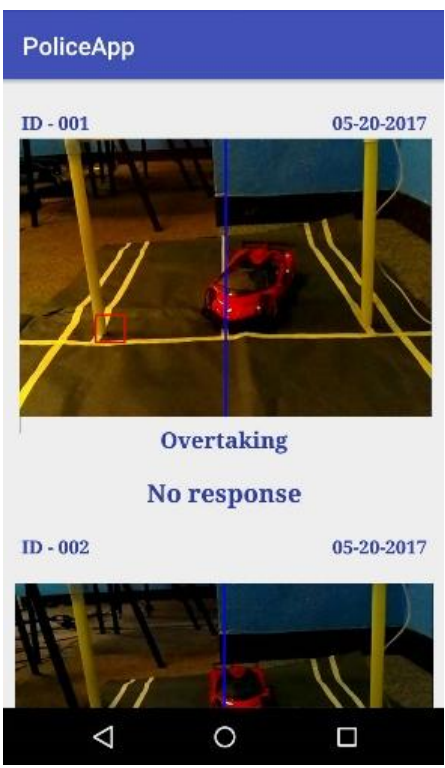

Fig 3: Message List

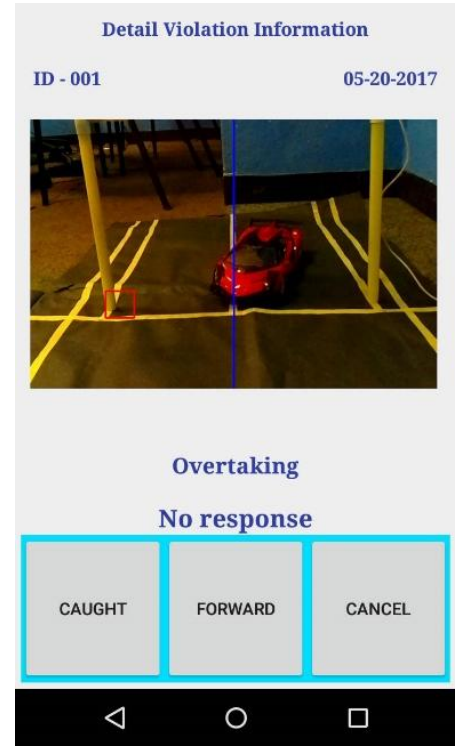

Fig 4: Message

As shown below in Fig 5 all the images that have captured by the analysis server can be checked by the web site. The user has to have an admin account in order to view.
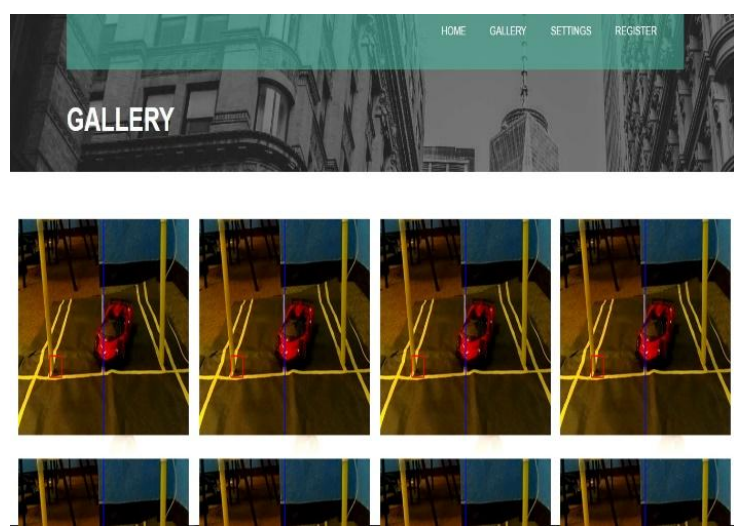

Fig 5: Hardware Circuit Diagram

\section{CONCLUSION AND FUTURE WORK}

Virtual police system is that a police officer can do his day to day job and reduce the traffic violations that happen daily at streets in Colombo area. Mobile phone usage in third world countries is playing a vital role for the enhancement so the police force will have the ability to control the traffic using virtual police system. There are many traffic violation detection systems but none of them doesn't serve the more violation detections than virtual police system.

The project "Virtual police" will be a better solution that uses cameras, ultrasonic sensors and image processing system to detect traffic violations. A police officer can get a visual image of the vehicle that violated the traffic law. Using this Virtual police system and virtual police mobile application police can control the traffic violations and day to day traffic jams that occur because of traffic violators. This system will be a reliable and accurate traffic violation detection system for police use.

In the process of the research project, the team had to deal with so many difficulties when developing the system. Some of the difficulties and limitations team had to face are listed down as followed, 
- Lack of resources of camera devices and its components.

- $\quad$ Lack of detection levels.

- Visibility of number plates in a traffic jam.

- Efficiency of the raspberry pi camera defects in different types of weather conditions.

As follows the research group decided to change some components of the system to change from some image processing techniques to ultrasonic sensor programming. The efficiency issues that had to face with image processing the group tended to find a better solution and had come up with ultrasonic to ensure to give better results.

Recommendations to those who are willing to develop this system further are as follows:

- Detecting violations at different weather conditions such as at night times

- $\quad$ Automate the fine tickets given to drivers.

- Police users to check logs of the messages and login times.

- Fine payment methods using VISA and Master credit or debit cards.

- Keep track of the vehicles that violates by recording there information.

- To automate the traffic light system with the use of android application.

As the research was limited to a specific time period the group was able to focus only a limited amount of components. In the future the group is willing to do more researches and develop more methods that will be included to the raspberry pi as well as to the android application and web applications.

\section{ACKNOWLEDGEMENT}

The research team take this opportunity to sincerely acknowledge the individuals and groups who have provided guidance and assistance throughout the project, First and foremost we would like to thank Dr. Yasas Jayaweera, the Academic Head of Sri Lanka Institute of Sri Lanka (SLIIT) $13^{\text {th }}$ floor. Finally we would like to acknowledge with gratitude, the great amount of support and advice given by all our family members and friends as well as all the other individuals and groups that gave a helping hand whose names were not mentioned.

\section{REFERENCES}

[1] "Implementing Lane Departure Warning System on Raspberry Pi", University Tunku Abdul Rahman, May 2016, [Online] Available: www.eprints.utar.edu.my/ 1950/1/CN-2016-1308318-1.pdf [Accessed 20-JULY2016]
[2] "A Real-Time Vision System for Night time Vehicle Detection and Traffic Surveillance", IEEE transactions on industrial electronics, Volume-58, No-5, May 2011, [Online] Available: https://ir.nctu.edu.tw/bitstream/ 11536/8964/1/000289478000054.pdf [Accessed: 18July2016]

[3] "Morphological Image Processing Approach of Vehicle Detection for Real-Time Traffic Analysis", Science International Journal of Computer Science and Engineering, Volume-2, Issue-5, May 2014. [Online] Available: http://www.ijcseonline.org/pub_paper/IJCSE00312.pdf [Accessed: 14-JULY-2016]

[4] "Development of an automated Red Light Violation Detection System (RLVDS) for Indian vehicles", IEEE National Conference on Computing and Communication Systems, COCOSYS-09, CS11 [Online] Available: https://arxiv.org/ftp/arxiv/papers/1003/1003.6052.pdf [Accessed: 14-JULY-2016]

[5] "Vehicle Detection under Day and Night Illumination", Proc. of ISCS-IIA99, May 2016, [Online] Available: http://imagelab.ing.unimore.it/pubblicazioni/pubblicazio ni/iia1999.pdf [Accessed 21JULY-2016]

[6] "A real-time computer vision system for vehicle tracking and traffic surveillance", Transportation Research Part C 6, CA 94720-1776, USA, Dec 1998, [Online] Available: http://people.eecs.berkeley.edu/ malik/papers/coiffmanB MM.pdf [Accessed: 21-July2016]

[7] "Automated Driving Vehicle Using Image Processing", International Journal of Computer Science and Engineering, Volume-2, Issue-4, April 2014,[Online] Available: http://www.ijcseonline.org/pub_paper/IJCSE00220.pdf [Accessed: 19-JULY-2016]

[8] Handaric R. et al. "An Image Recognition Software Tool for Car Identification Based on License Plate", Transylvania University of Braşov Series I: Engineering Sciences, Volume-5 (54) No. 1, 2012, [Online] Available: http://rs.unitbv.ro/BU2012/Series\%20I/BULETIN\%20I \%20PDF/Handaric_R.pdf [Accessed 19-JULY-2016]

[9] "Object Detection in Image Processing Using Edge Detection Techniques", and IOSR Journal of Engineering, ISSN (e): 2250-3021, ISSN (p): 2278-8719 Vol. 04, Issue 03, March 2014 [Accessed: 21-JULY2016]

[10] "Hybrid Parking Detection", United States Patent Application Publication, Patent No. US20150117705A1, April 2015 [Accessed: 19JULY2016] 